\title{
Taming PITCHf/x Data with XML2R and pitchRx
}

by Carson Sievert

\begin{abstract}
XML2R is a framework that reduces the effort required to transform XML content into tables in a way that preserves parent to child relationships. pitchRx applies XML2R's grammar for XML manipulation to Major League Baseball Advanced Media (MLBAM)'s Gameday data. With pitchRx, one can easily obtain and store Gameday data in a remote database. The Gameday website hosts a wealth of XML data, but perhaps most interesting is PITCHf/x. Among other things, PITCHf/x data can be used to recreate a baseball's flight path from a pitcher's hand to home plate. With pitchRx, one can easily create animations and interactive 3D scatterplots of the baseball's flight path. PITCHf/x data is also commonly used to generate a static plot of baseball locations at the moment they cross home plate. These plots, sometimes called strike-zone plots, can also refer to a plot of event probabilities over the same region. pitchRx provides an easy and robust way to generate strike-zone plots using the ggplot2 package.
\end{abstract}

\section{Introduction}

What is PITCHf/x?

PITCHf/ $x$ is a general term for a system that generates a series of 3D measurements of a baseball's path from a pitcher's hand to home plate (Alt and White, 2008). ${ }^{1}$ In an attempt to estimate the location of the ball at any time point, a quadratic regression model with nine parameters (defined by the equations of motion for constant linear acceleration) is fit to each pitch. Studies with access to the actual measurements suggest that this model is quite reasonable - especially for non-knuckleball pitches (Nathan, 2008). That is, the fitted path often provides a reasonable estimate (within a couple of inches) of the actual locations. Unfortunately, only the parameter estimates are made available to the public. The website that provides these estimates is maintained by MLBAM and hosts a wealth of other baseball related data used to inform MLB's Gameday webcast service in near real time.

\section{Why is PITCHf/x important?}

On the business side of baseball, using statistical analysis to scout and evaluate players has become mainstream. When PITCHf/x was first introduced, (DiMeo, 2007) proclaimed it as,

"The new technology that will change statistical analysis [of baseball] forever."

PITCHf/ $x$ has yet to fully deliver this claim, partially due to the difficulty in accessing and deriving insight from the large amount of complex information. By providing better tools to collect and visualize this data, pitchRx makes PITCHf/x analysis more accessible to the general public.

\section{PITCHf/x applications}

PITCHf/ $x$ data is and can be used for many different projects. It can also complement other baseball data sources, which poses an interesting database management problem. Statistical analysis of PITCHf/ $x$ data and baseball in general has become so popular that it has helped expose statistical ideas and practice to the general public. If you have witnessed television broadcasts of MLB games, you know one obvious application of PITCHf/x is locating pitches in the strike-zone as well as recreating flight trajectories, tracking pitch speed, etc. Some on-going statistical research related to PITCHf/x includes: classifying pitch types, predicting pitch sequences, and clustering pitchers with similar tendencies (Pane et al., 2013).

\section{Contributions of pitchRx and XML2R}

pitchRx has two main focuses (Sievert, 2014b). The first focus is to provide easy access to Gameday data. Not only is pitchRx helpful for collecting this data in bulk, but it has served as a helpful teaching and research aide (http://baseballwithr. wordpress.com/ is one such example). Methods

\footnotetext{
${ }^{1} \mathrm{~A}$ pitcher throws a ball to the opposing batter, who stands besides home plate and tries to hit the ball into the field of play.
} 
for collecting Gameday data existed prior to pitchRx; however, these methods are not easily extensible and require juggling technologies that may not be familiar or accessible (Fast, 2007). Moreover, these working environments are less desirable than $\mathrm{R}$ for data analysis and visualization. Since pitch $\mathbf{R x}$ is built upon XML2R's united framework, it can be easily modified and/or extended (Sievert, 2014a). For this same reason, pitchRx serves as a model for building customized XML data collection tools with XML2R.

The other main focus of pitchRx is to simplify the process creating popular PITCHf/x graphics. Strike-zone plots and animations made via pitchRx utilize the extensible ggplot2 framework as well as various customized options (Wickham, 2009). ggplot2 is a convenient framework for generating strike-zone plots primarily because of its facet schema which allows one to make visual comparisons across any combination of discrete variable(s). Interactive 3D scatterplots are based on the rgl package and useful for gaining a new perspective on flight trajectories (Adler et al.).

\section{Getting familiar with Gameday}

Gameday data is hosted and made available for free thanks to MLBAM via http://gd2.mlb.com/ components/game/mlb/. ${ }^{2}$ From this website, one can obtain many different types of data besides PITCHf/x. For example, one can obtain everything from structured media metadata to insider tweets. In fact, this website's purpose is to serve data to various $h t t p: / / \mathrm{ml}$ b. com web pages and applications. As a result, some data is redundant and the format may not be optimal for statistical analysis. For these reasons, the scrape function is focused on retrieving data that is useful for PITCHf/x analysis and providing it in a convenient format for data analysis.

Navigating through the MLBAM website can be overwhelming, but it helps to recognize that a homepage exists for nearly every day and every game. For example, http://gd2. mlb. com/components/ game/mlb/year_2011/month_02/day_26/ displays numerous hyperlinks to various files specific to February 26th, 2011. On this page is a hyperlink to miniscoreboard.xml which contains information on every game played on that date. This page also has numerous hyperlinks to game specific pages. For example, gid_2011_02_26_phimlb_nyamlb_1/ points to the homepage for that day's game between the NY Yankees and Philadelphia Phillies. On this page is a hyperlink to the players.xml file which contains information about the players, umpires, and coaches (positions, names, batting average, etc.) coming into that game.

Starting from a particular game's homepage and clicking on the inning/ directory, we should see another page with links to the inning_all.xml file and the inning_hit.xml file. If it is available, the inning_all.xml file contains the PITCHf/x data for that game. It's important to note that this file won't exist for some games, because some games are played in venues that do not have a working PITCHf/x system in place. This is especially true for preseason games and games played prior to the 2008 season when the PITCHf/x system became widely adopted. ${ }^{3}$ The inning_hit. xml files have manually recorded spatial coordinates of where a home run landed or where the baseball made initial contact with a defender after it was hit into play.

The relationship between these XML files and the tables returned by scrape is presented in Table 1. The pitch table is extracted from files whose name ends in inning_all.xml. This is the only table returned by scrape that contains data on the pitch-by-pitch level. The atbat, runner, action and hip tables from this same file are commonly labeled somewhat ambiguously as play-by-play data. The player, coach, and umpire tables are extracted from players. xml and are classified as game-by-game since there is one record per person per game. Figure 1 shows how these tables can be connected to one another in a database setting. The direction of the arrows represent a one to possibly many relationship. For example, at least one pitch is thrown for each at bat (that is, each bout between pitcher and batter) and there are numerous at bats within each game.

In a rough sense, one can relate tables returned by scrape back to XML nodes in the XML files. For convenience, some information in certain XML nodes are combined into one table. For example, information gleaned from the 'top', 'bottom', and 'inning' XML nodes within inning_all.xml are included as inning and inning_side fields in the pitch, po, atbat, runner, and action tables. This helps reduce the burden of merging many tables together in order to have inning information on the play-by-play and/or pitch-by-pitch level. Other information is simply ignored simply because it is redundant. For example, the 'game' node within the players. xml file contains information that can be recovered from the game table extracted from the miniscoreboard.xml file. If the reader wants a more detailed explanation of fields in these tables, Marchi and Albert (2013) provide nice overview.

\footnotetext{
${ }^{2}$ Please be respectful of this service and store any information after you extract it instead of repeatedly querying the website. Before using any content from this website, please also read the copyright.

${ }^{3}$ In this case, scrape will print "failed to load HTTP resource" in the R console (after the relevant file name) to indicate that no data was available.
} 


\begin{tabular}{cccc}
\hline $\begin{array}{c}\text { Source file } \\
\text { suffix }\end{array}$ & $\begin{array}{c}\text { Information } \\
\text { level }\end{array}$ & XML nodes & $\begin{array}{c}\text { Tables returned } \\
\text { by scrape }\end{array}$ \\
\hline miniscoreboard.xml & game-by-game & $\begin{array}{c}\text { games, game, } \\
\text { game_media, media }\end{array}$ & game, media \\
\hline players.xml & game-by-game & $\begin{array}{c}\text { game, team, player, } \\
\text { coach, umpire }\end{array}$ & $\begin{array}{c}\text { player, coach, } \\
\text { umpire }\end{array}$ \\
\hline inning_all.xml & $\begin{array}{c}\text { play-by-play, } \\
\text { pitch-by-pitch }\end{array}$ & $\begin{array}{c}\text { game, inning, bottom, top, } \\
\text { atbat, po, pitch, runner, action }\end{array}$ & $\begin{array}{c}\text { atbat, po, pitch, } \\
\text { runner, action }\end{array}$ \\
\hline inning_hit.xml & play-by-play & hitchart, hip & hip \\
\hline
\end{tabular}

Table 1: Structure of PITCHf/x and related Gameday data sources accessible to scrape

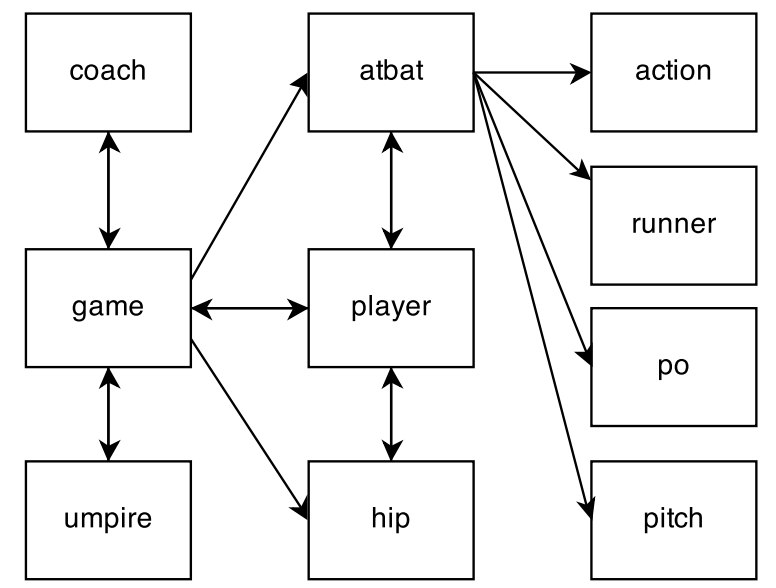

Figure 1: Table relations between Gameday data accessible via scrape. The direction of the arrows indicate a one to possibly many relationship.

\section{Introducing XML2R}

XML2R adds to the CRAN Task View on Web Technologies and Services by focusing on the transformation of XML content into a collection of tables. Compared to a lower-level API like the XML package, it can significantly reduce the amount of coding and cognitive effort required to perform such a task. In contrast to most higher-level APIs, it does not make assumptions about the XML structure or its source. Although XML2R works on any structure, performance and user experience are enhanced if the content has an inherent relational structure. XML2R's novel approach to XML data collection breaks down the transformation process into a few simple steps and allows the user to decide how to apply those steps.

The next few sections demonstrate how pitchRx leverages XML2R in order to produce a collection of tables from inning_all.xml files. A similar approach is used by pitchRx: : scrape to construct tables from the other Gameday files in Table 1. In fact, XML2R has also been used in the R package bbscrapeR which collects data from nba.com and wnba.com.

\section{Constructing file names}

Sometimes the most frustrating part of obtaining data in bulk off of the web is finding the proper collection of URLs or file names of interest. Since files on the Gameday website are fairly well organized, the makeUrls function can be used to construct urls that point to every game's homepage within a window of dates.

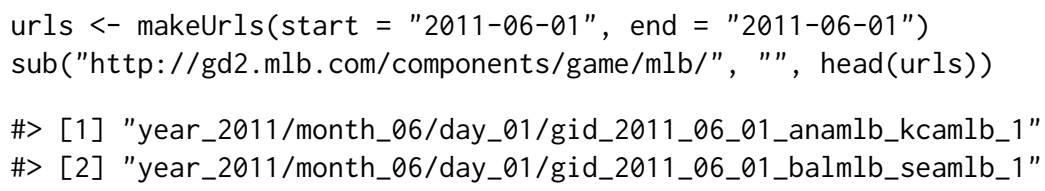


\#> [3] "year_2011/month_06/day_01/gid_2011_06_01_chamlb_bosmlb_1"
\#> [4] "year_2011/month_06/day_01/gid_2011_06_01_clemlb_tormlb_1"
\#> [5] "year_2011/month_06/day_01/gid_2011_06_01_colmlb_lanmlb_1"
\#> [6] "year_2011/month_06/day_01/gid_2011_06_01_flomlb_arimlb_1"

\section{Extracting observations}

Once we have a collection of XML files, the next step is to parse the content into a list of observations. An observation is technically defined as a matrix with one row and some number of columns. The columns are defined by XML attributes and the XML value (if any) for a particular XML lineage. The name of each observation tracks the XML hierarchy so observations can be grouped together in a sensible fashion at a later point.

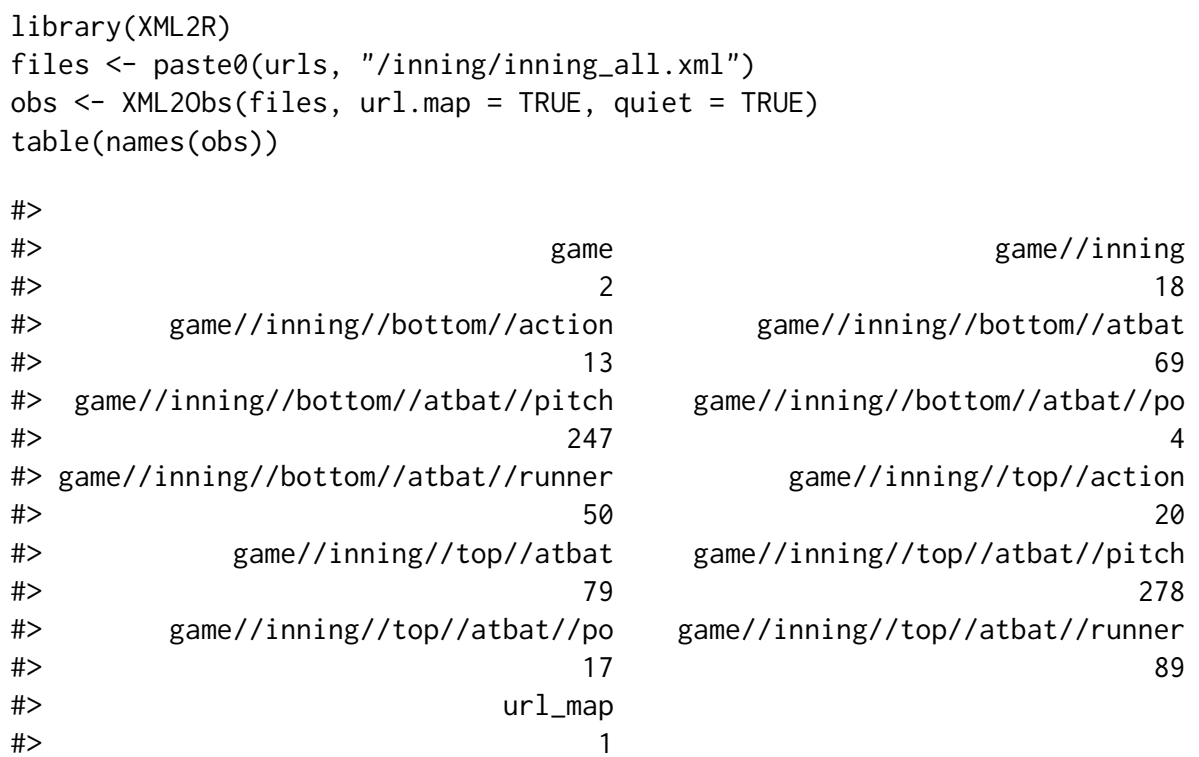

This output tells us that 247 pitches were thrown in the bottom inning and 278 were thrown in the top inning on June 1st, 2011. Also, there are 12 different levels of observations. The list element named url_map is not considered an observation and was included since url.map = TRUE. This helps avoid repeating long file names in the url_key column which tracks the mapping between observations and file names.

obs[1]

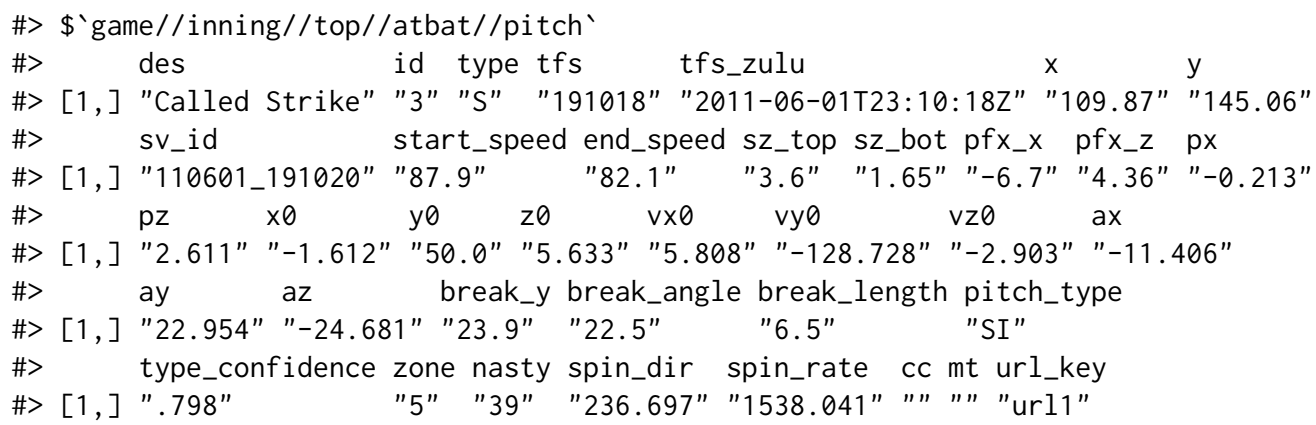

\section{Renaming observations}

Before grouping observations into a collection tables based on their names, one may want to re_name observations. Observations with names 'game//inning//bottom//atbat' and 'game//inning//top//atbat ' should be included in the same table since they share XML attributes (in other words, the observations share variables).

tmp <- re_name(obs, equiv = c("game//inning//top//atbat",

"game//inning//bottom//atbat"), diff.name = "inning_side") 
By passing these names to the equiv argument, re_name determines the difference in the naming scheme and suppresses that difference. In other words, observation names that match the equiv argument will be renamed to 'game//inning//atbat'. The information removed from the name is not lost; however, as a new column is appended to the end of each relevant observation. For example, notice how the inning_side column contains the part of the name we just removed:

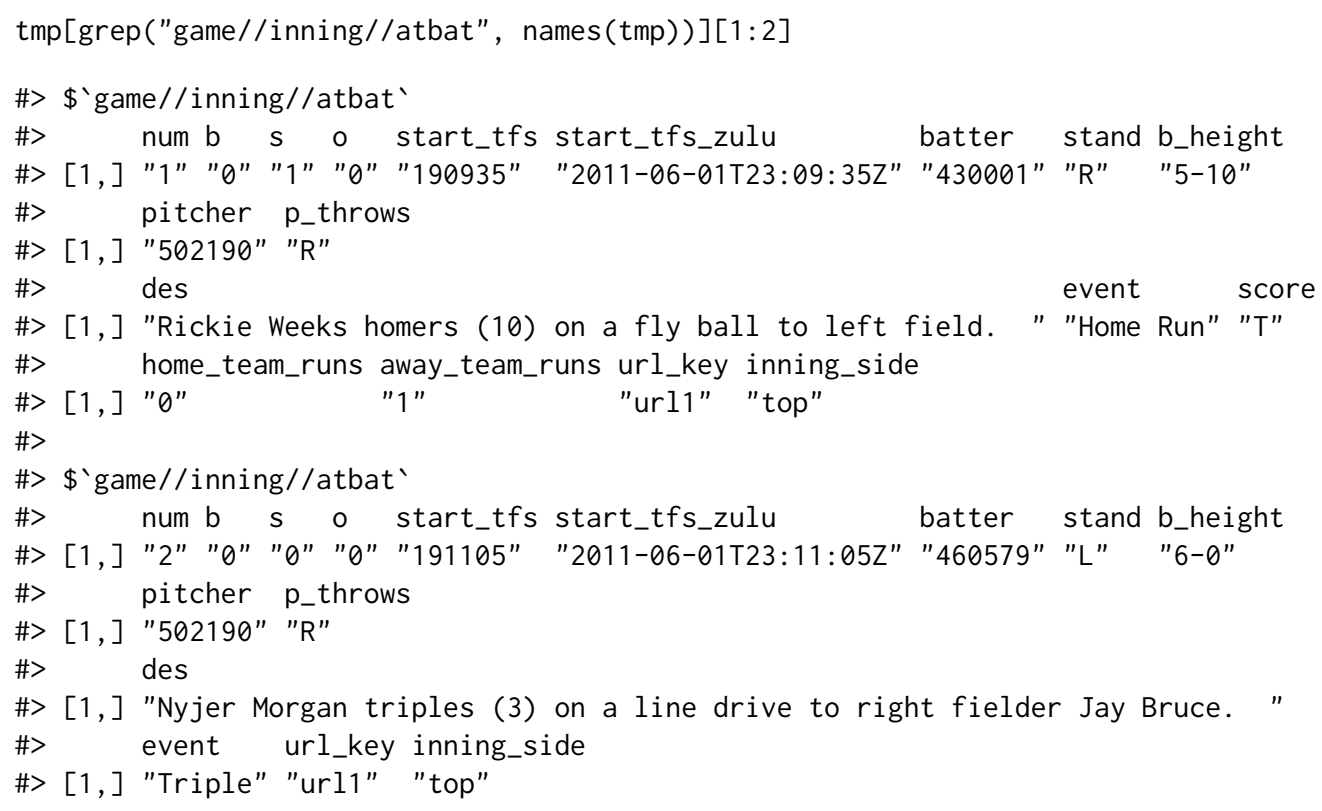

For similar reasons, other observation name pairs are renamed in a similar fashion.

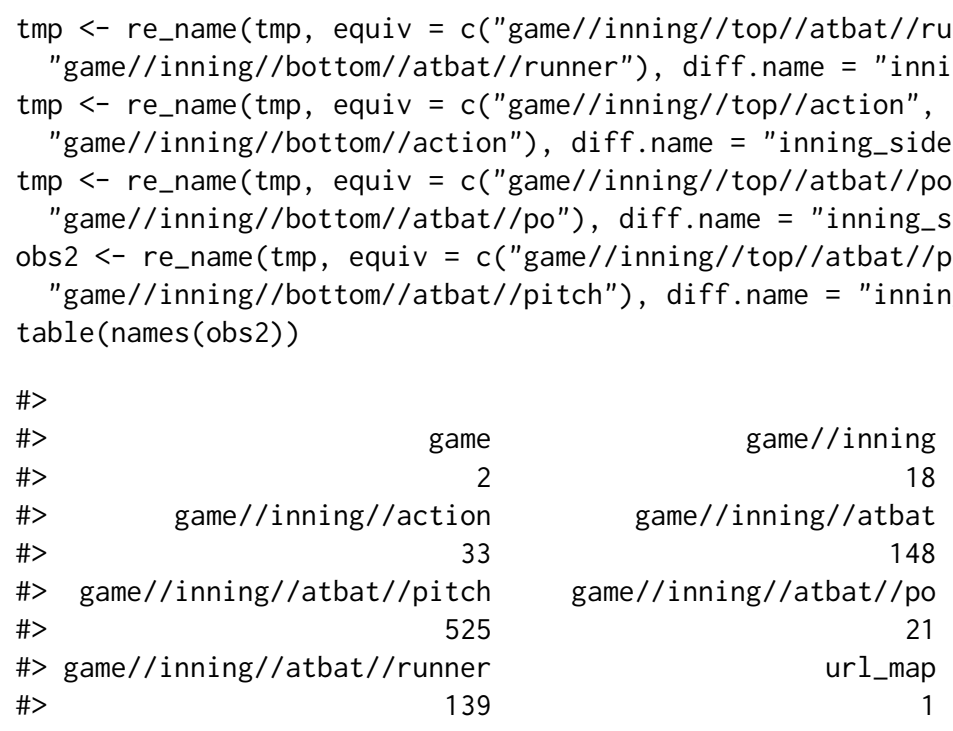

\section{Linking observations}

After all that renaming, we now have 7 different levels of observations. Let's examine the first three observations on the game//inning level:

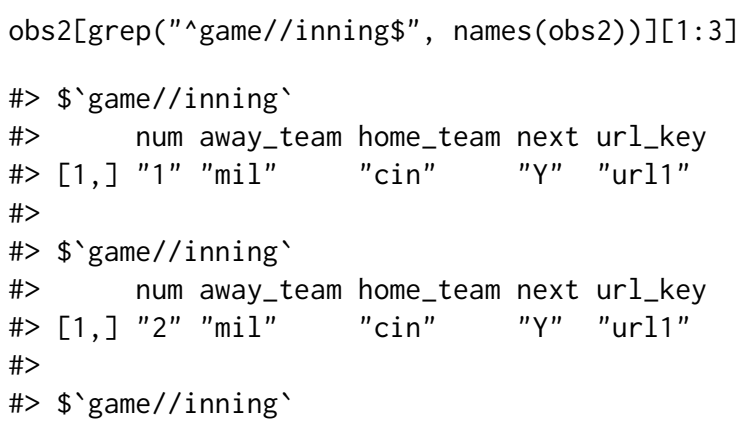


\#> num away_team home_team next url_key

\#> $[1$,$] "3" "mil" "cin" "Y" "url1"$

Before grouping observations into tables, it is usually important preserve the parent-to-child relationships in the XML lineage. For example, one may want to map a particular pitch back to the inning in which it was thrown. Using the add_key function, the relevant value of num for game//inning observations can be recycled to its XML descendants.

obswkey <- add_key(obs2, parent = "game//inning", recycle = "num", key. name = "inning")

\#> A key for the following children will be generated for the game//inning node:

\#> game//inning//atbat//pitch

\#> game//inning//atbat//runner

\#> game//inning//atbat

\#> game//inning//atbat//po

\#> game//inning//action

As it turns out, the away_team and home_team columns are redundant as this information is embedded in the url column. Thus, there is only one other informative attribute on this level which is next. By recycling this value among its descendants, we remove any need to retain a game//inning table.

obswkey <- add_key (obswkey, parent = "game//inning", recycle = "next")

\#> A key for the following children will be generated for the game//inning node:

\#> game//inning//atbat//pitch

\#> game//inning//atbat//runner

\#> game//inning//atbat

\#> game//inning//atbat//po

\#> game//inning//action

It is also imperative that we can link a pitch, runner, or po back to a particular atbat. This can be done as follows:

obswkey <- add_key (obswkey, parent = "game//inning//atbat", recycle = "num")

\#> A key for the following children will be generated for the game//inning//atbat node:

\#> game//inning//atbat//pitch

\#> game//inning//atbat//runner

\#> game//inning//atbat//po

\section{Collapsing observations}

Finally, we are in a position to pool together observations that have a common name. The collapse_obs function achieves this by row binding observations with the same name together and returning a list of matrices. Note that collapse_obs does not require that observations from the same level to have the same set of variables in order to be bound into a common table. In the case where variables are missing, NAs will be inserted as values.

tables <- collapse_obs(obswkey)

\#As mentioned before, we do not need the 'inning' table

tables <- tables[!grepl("^game//inning\$", names(tables))]

table.names <- c("game", "action", "atbat", "pitch", "po", "runner")

tables <- setNames (tables, table.names)

head(tables[["runner"]])

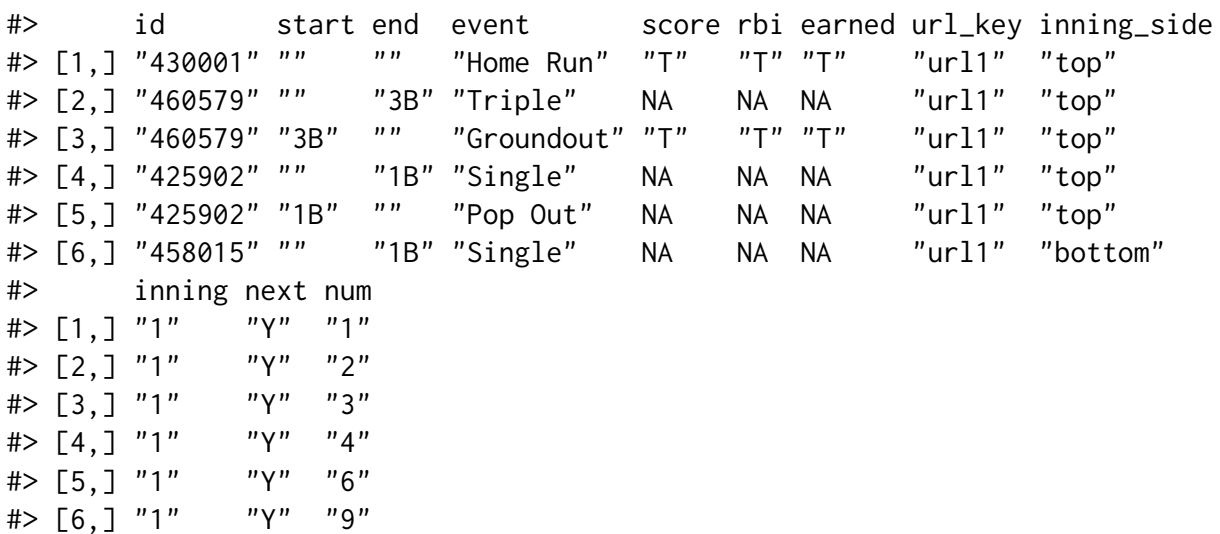




\section{Collecting Gameday data with pitchRx}

The main scraping function in pitchRx, scrape, can be used to easily obtain data from the files listed in Table 1. In fact, any combination of these files can be queried using the suffix argument. In the example below, the start and end arguments are also used so that all available file types for June 1st, 2011 are queried.

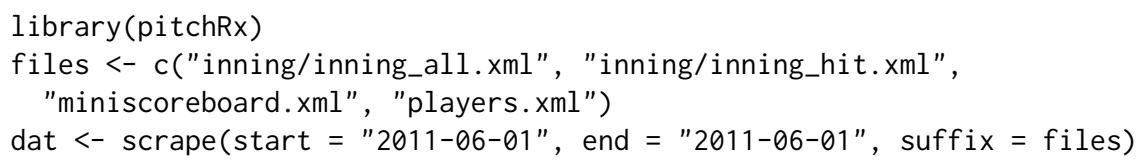

The game.ids option can be used instead of start and end to obtain an equivalent dat object. This option can be useful if the user wants to query specific games rather than all games played over a particular time span. When using this game. ids option, the built-in gids object, is quite convenient.

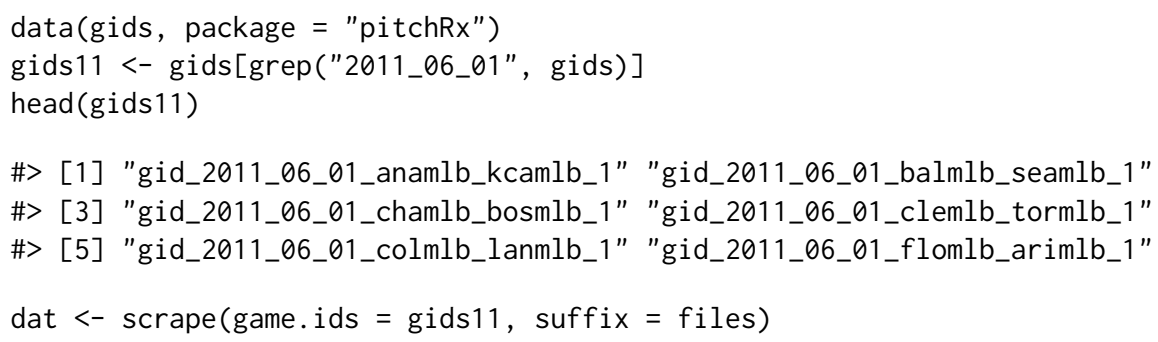

The object dat is a list of data frames containing all data available for June 1st, 2011 using scrape. The list names match the table names provided in Table 1. For example, dat $\$ a$ tbat is data frame with every at bat on June 1st, 2011 and dat\$pitch has information related to the outcome of each pitch (including PITCHf/x parameters). The object. size of dat is nearly 300MB. Multiplying this number by 100 days exceeds the memory of most machines. Thus, if a large amount of data is required, the user should exploit the R database interface (R Special Interest Group on Databases, 2013).

\section{Storing and querying Gameday data}

Since PITCHf/x data can easily exhaust memory, one should consider establishing a database instance before using scrape. By passing a database connection to the connect argument, scrape will try to create (and/or append to existing) tables using that connection. If the connection fails for some reason, tables will be written as csv files in the current working directory. The benefits of using the connect argument includes improved memory management which can greatly reduce run time. connect will support a MySQL connection, but creating a SQLite database is quite easy with dplyr (Wickham and Francois, 2014).

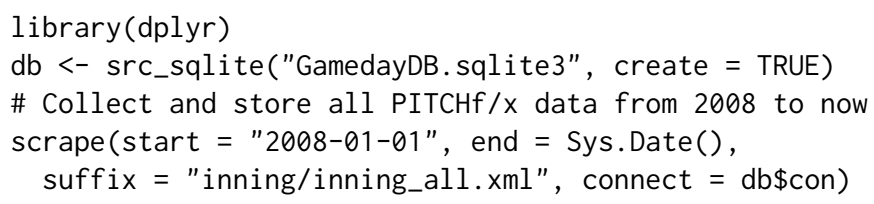

In the later sections, animations of four-seam and cut fastballs thrown by Mariano Rivera and Phil Hughes during the 2011 season are created. In order to obtain the data for those animations, one could query $\mathrm{db}$ which now has PITCHf/x data from 2008 to date. This query requires criteria on: the pitcher_name field (in the atbat table), the pitch_type field (in the pitch table), and the date field (in both tables). To reduce the time required to search those records, one should create an index on each of these three fields.

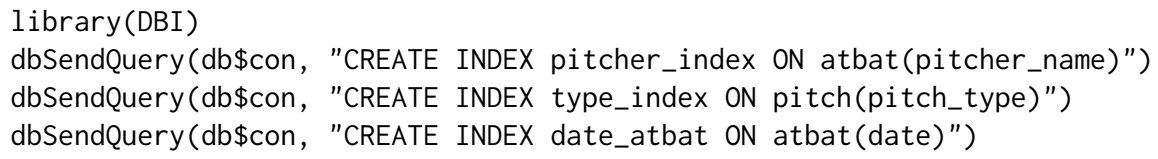

As a part of our query, we'll have to join the atbat table together with the pitch table. For this task, the gameday_link and num fields are helpful since together they provide a way to match pitches with at bats. For this reason, a multi-column index on the gameday_link and num fields will further reduce run time of the query. 
dbSendQuery (db\$con, 'CREATE INDEX pitch_join ON pitch(gameday_link, num)')

dbSendQuery(db\$con, 'CREATE INDEX atbat_join ON atbat(gameday_link, num)')

Although the query itself could be expressed entirely in SQL, dplyr's grammar for data manipulation (which is database agnostic) can help to simplify the task. In this case, at . bat is a tabular representation of the remote atbat table restricted to cases where Rivera or Hughes was the pitcher. That is, at. bat does not contain the actual data, but it does contain the information necessary to retrieve it from the database.

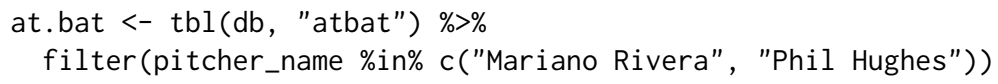

Similarly, fbs is a tabular representation of the pitch table restricted to four-seam (FF) and cut fastballs (FC).

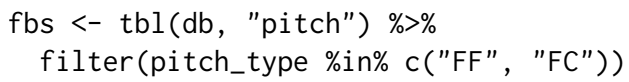

An inner_join of these two filtered tables returns a tabular representation of all four-seam and cut fastballs thrown by Rivera and Hughes. Before collect actually performs the database query and brings the relevant data into the R session, another restriction is added so that only pitches from 2011 are included.

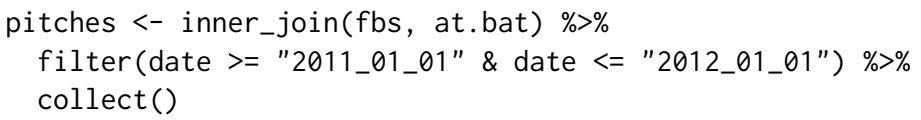

\section{Visualizing PITCHf/x}

\section{Strike-zone plots and umpire bias}

Amongst the most common PITCHf/x graphics are strike-zone plots. Such a plot has two axes and the coordinates represent the location of baseballs as they cross home plate. The term strike-zone plot can refer to either density or probabilistic plots. Density plots are useful for exploring what actually occurred, but probabilistic plots can help address much more interesting questions using statistical inference. Although probabilistic plots can be used to visually track any event probability across the strike-zone, their most popular use is for addressing umpire bias in a strike versus ball decision Green and Daniels (2014). The probabilistic plots section demonstrates how pitchRx simplifies the process behind creating such plots via a case study on the impact of home field advantage on umpire decisions.

In the world of sports, it is a common belief that umpires (or referees) have a tendency to favor the home team. PITCHf/x provides a unique opportunity to add to this discussion by modeling the probability of a called strike at home games versus away games. Specifically, conditioned upon the umpire making a decision at a specific location in the strike-zone, if the probability that a home pitcher receives a called strike is higher than the probability that an away pitcher receives a called strike, then there is evidence to support umpire bias towards a home pitcher.

There are many different possible outcomes of each pitch, but we can condition on the umpire making a decision by limiting to the following two cases. A called strike is an outcome of a pitch where the batter does not swing and the umpire declares the pitch a strike (which is a favorable outcome for the pitcher). A ball is another outcome where the batter does not swing and the umpire declares the pitch a ball (which is a favorable outcome for the batter). All decisions made between 2008 and 2013 can be obtained from $\mathrm{db}$ with the following query using dplyr.

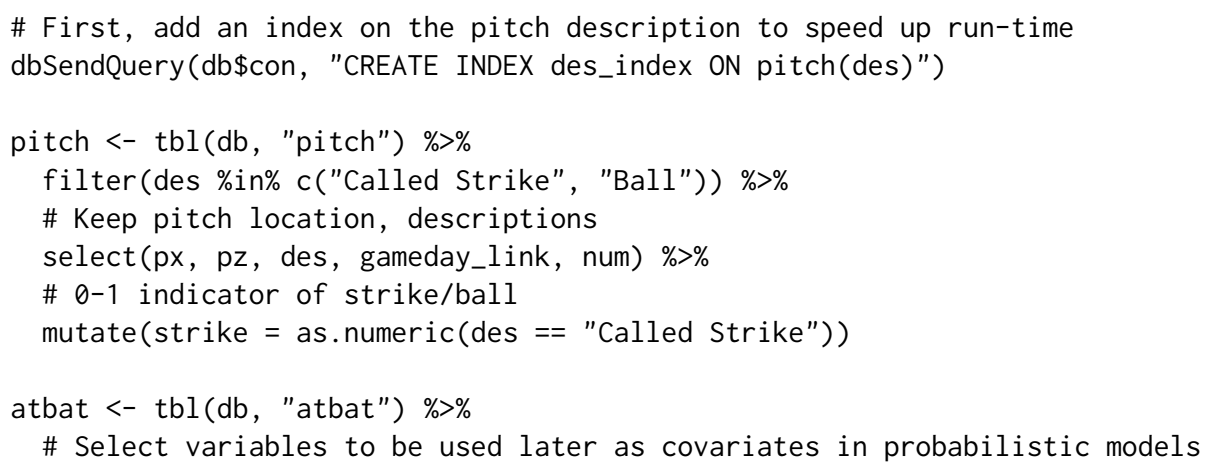


select(b_height, p_throws, stand, inning_side, date, gameday_link, num)

decisions <- inner_join(pitch, atbat) \%>\%

filter(date <= "2014_01_01") \%>\%

$\operatorname{collect}()$

\section{Density plots}

The decisions data frame contains data on over 2.5 million pitches thrown from 2008 to 2013. About a third of them are called strikes and two-thirds balls. Figure 2 shows the density of all called strikes. Clearly, most called strikes occur on the outer region of the strike-zone. Many factors could contribute to this phenomenon; which we will not investigate here.
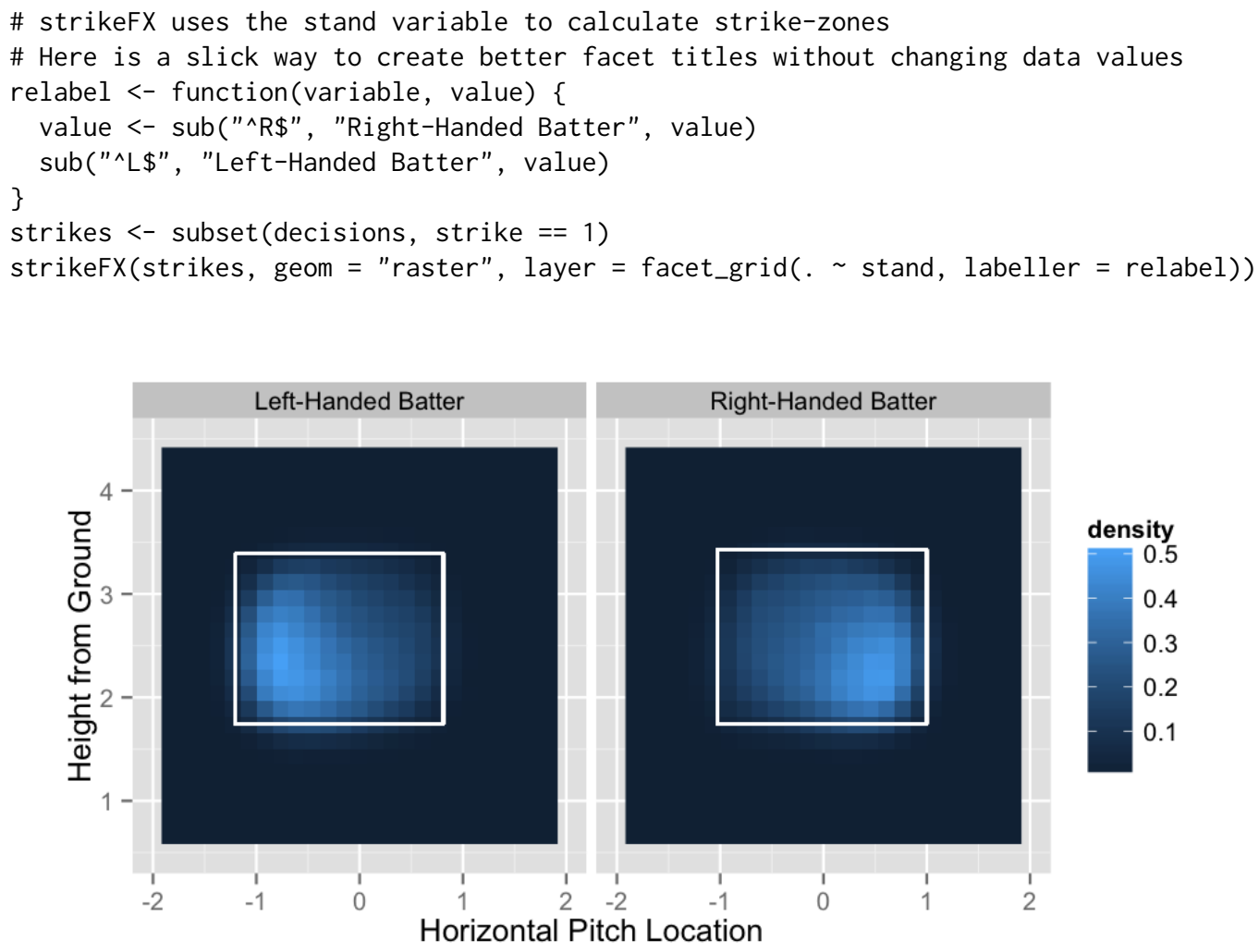

Figure 2: Density of called strikes for right-handed batters and left-handed batters (from 2008 to 2013).

Figure 2 shows one static rectangle (or strike-zone) per plot automatically generated by strikeFX. The definition of the strike-zone is notoriously ambiguous. As a result, the boundaries of the strikezone may be noticeably different in some situations. However, we can achieve a fairly accurate representation of strike-zones using a rectangle defined by batters' average height and stance (Fast, 2011). As Figure 4 reinforces, batter stance makes an important difference since the strike-zone seems to be horizontally shifted away from the batter. The batter's height is also important since the strike-zone is classically defined as approximately between the batter's knees and armpits.

Figure 2 has is one strike-zone per plot since the layer option contains a ggplot 2 argument that facets according to batter stance. Facet layers are a powerful tool for analyzing PITCHf/x data because they help produce quick and insightful comparisons. In addition to using the layer option, one can add layers to a graphic returned by strikeFX using ggplot2 arithmetic. It is also worth pointing out that Figure 2 could have been created without introducing the strikes data frame by using the density 1 and density 2 options.

strikeFX(decisions, geom = "raster", density1 = list (des = "Called Strike"), density2 $=$ list $($ des $=$ "Called Strike") $)+$ facet_grid $($. stand, labeller $=$ relabel $)$

In general, when density 1 and density 2 are identical, the result is equivalent to subsetting the data frame appropriately beforehand. More importantly, by specifying different values for density 1 and density2, differenced densities are easily generated. In this case, a grid of density estimates for 
density 2 are subtracted from the corresponding grid of density estimates for density 1 . Note that the default NULL value for either density option infers that the entire data set defines the relevant density. Thus, if density2 was NULL (when density1 = list(des = 'Called Strike')), we would obtain the density of called strikes minus the density of both called strikes and balls. In Figure 3, we define density 1 as called strikes and define density 2 as balls. As expected, we see positive density values (in blue) inside the strike-zone and negative density values (in red) outside of the strike-zone.
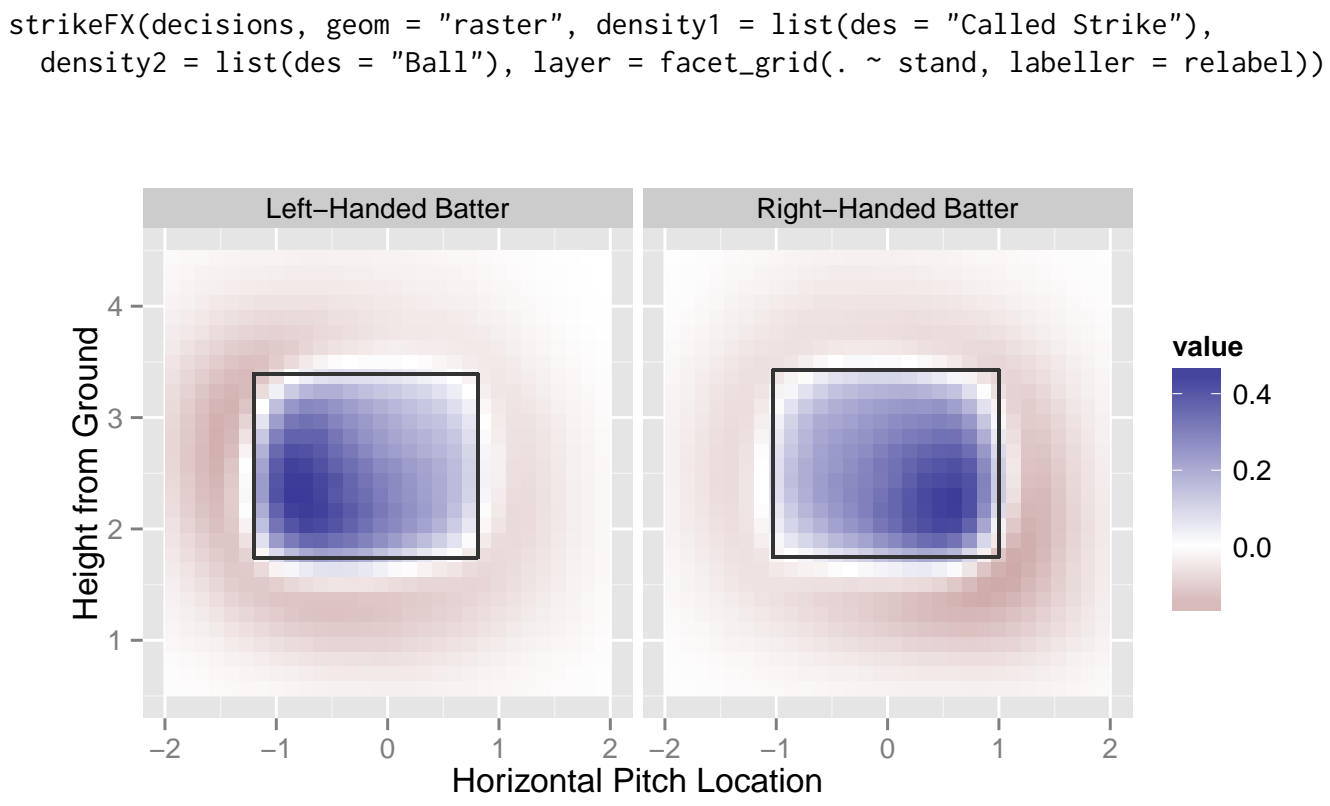

Figure 3: Density of called strikes minus density of balls for both right-handed batters and left-handed batters (from 2008 to 2013). The blue region indicates a higher frequency of called strikes and the red region indicates a higher frequency of balls.

These density plots are helpful for visualizing the observed frequency of events; however, they are not very useful for addressing our umpire bias hypothesis. Instead of looking simply at the density, we want to model the probability of a strike called at each coordinate given the umpire has to make a decision.

\section{Probabilistic plots}

There are many approaches to probabilistic modeling over a two dimensional spatial region. Since our response is often categorical, generalized additive models (GAMs) is a popular and desirable approach to modeling events over the strike-zone (Mills, 2010). There are numerous R package implementations of GAMs, but the bam function from the mgcv package has several desirable properties (Wood, 2006). Most importantly, the smoothing parameter can be estimated using several different methods. In order to have a reasonable estimate of the smooth 2D surface, GAMs require fairly large amount of observations. As a result, run time can be an issue - especially when modeling 2.5 million observations! Thankfully, the bam function has a cluster argument which allows one to distribute computations across multiple cores using the built in parallel package.

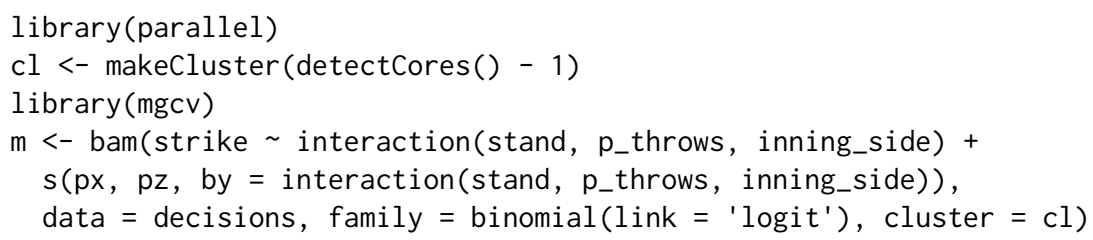

This formula models the probability of a strike as a function of the baseball's spatial location, the batter's stance, the pitcher's throwing arm, and the side of the inning. Since home pitchers always pitch during the top of the inning, inning_side also serves as an indication of whether a pitch is thrown by a home pitcher. In this case, the interaction function creates a factor with eight different levels since every input factor has two levels. Consequently, there are 8 different levels of smooth surfaces over the spatial region defined by px and pz. 
The fitted model $\mathrm{m}$ contains a lot of information which strikeFX uses in conjunction with any ggplot2 facet commands to infer which and how surfaces should be plotted. In particular, the var. summary is used to identify model covariates, as well their default conditioning values. In our case, the majority of decisions are from right-handed pitchers and the top of the inning. Thus, the default conditioning values are "top" for inning_side and " $R$ " for $p_{-}$throws. If different conditioning values are desired, var . summary can be modified accordingly. To demonstrate, Figure 4 shows 2 of the 8 possible surfaces that correspond to a right-handed away pitcher.

away <- list (inning_side = factor("bottom", levels = c("top", "bottom")))

$m \$$ var. summary <- modifyList ( $m \$ v a r$. summary, away)

strikeFX(decisions, model $=\mathrm{m}$, layer $=$ facet_grid $(. \sim$ stand, labeller $=$ relabel $)$ )

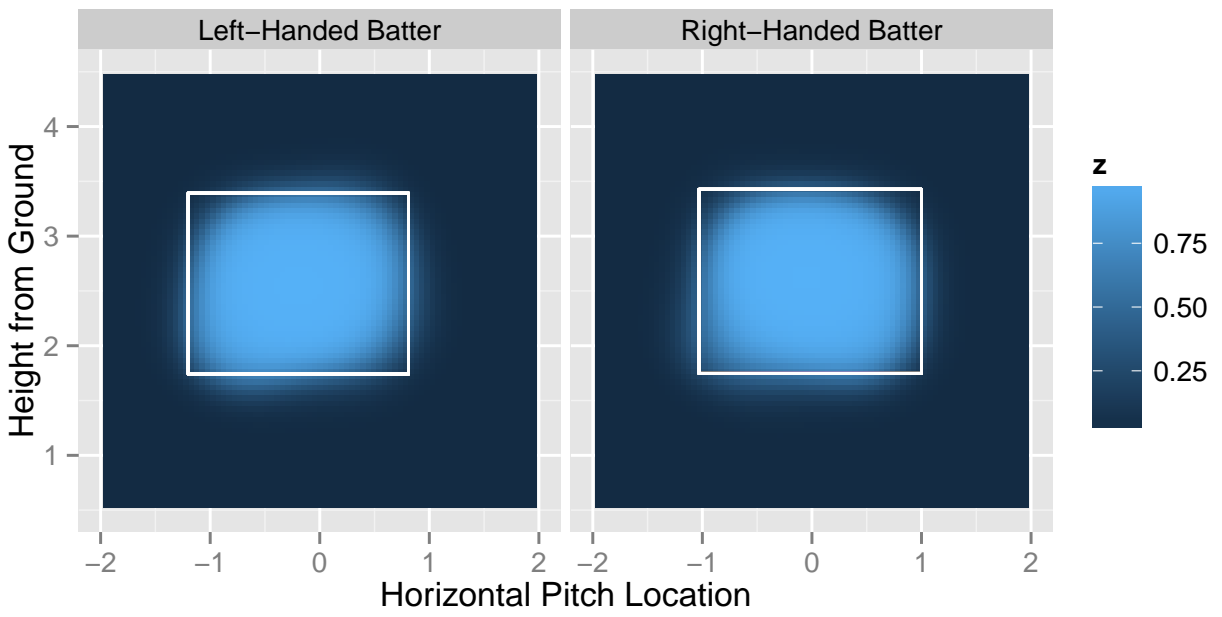

Figure 4: Probability that a right-handed away pitcher receives a called strike (provided the umpire has to make a decision). Plots are faceted by the handedness of the batter.

Using the same intuition exploited earlier to obtain differenced density plots, we can easily obtain differenced probability plots. To obtain Figure 5, we simply add p_throws as another facet variable and inning_side as a differencing variable. In this case, conditioning values do not matter since every one of the 8 surfaces are required in order to produce Figure 5.

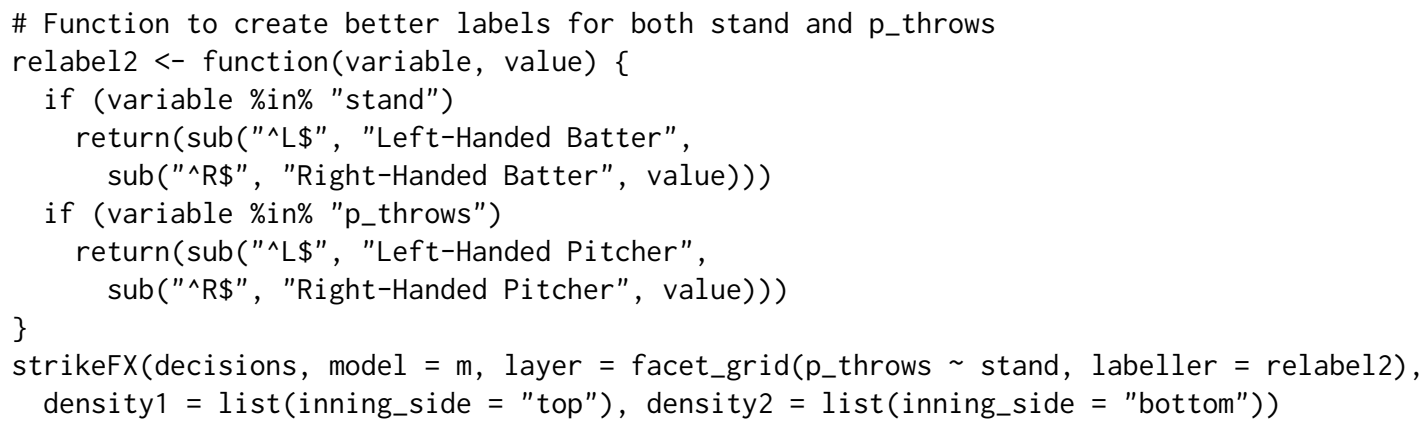

The four different plots in Figure 5 represent the four different combination of values among $p_{-}$throws and stand. In general, provided that a pitcher throws to a batter in the blue region, the pitch is more likely to be called a strike if the pitcher is on their home turf. Interestingly, there is a well-defined blue elliptical band around the boundaries of the typical strike-zone. Thus, home pitchers are more likely to receive a favorable call - especially when the classification of the pitch is in question. In some areas, the home pitcher has up to a 6 percent higher probability of receiving a called strike than an away pitcher. The subtle differences in spatial patterns across the different values of $p_{-}$throws and stand are interesting as well. For instance, pitching at home has a large positive impact for a left-handed pitcher throwing in the lower inside portion of the strike-zone to a right-handed batter, but the impact seems negligible in the mirror opposite case. Differenced probabilistic densities are clearly an interesting visual tool for analyzing PITCHf/x data. With strikeFX, one can quickly and easily make all sorts of visual comparisons for various situations. In fact, one can explore and compare the probabilistic structure of any well-defined event over a strike-zone region (for example, the probability a batter reaches base) using a similar approach. 


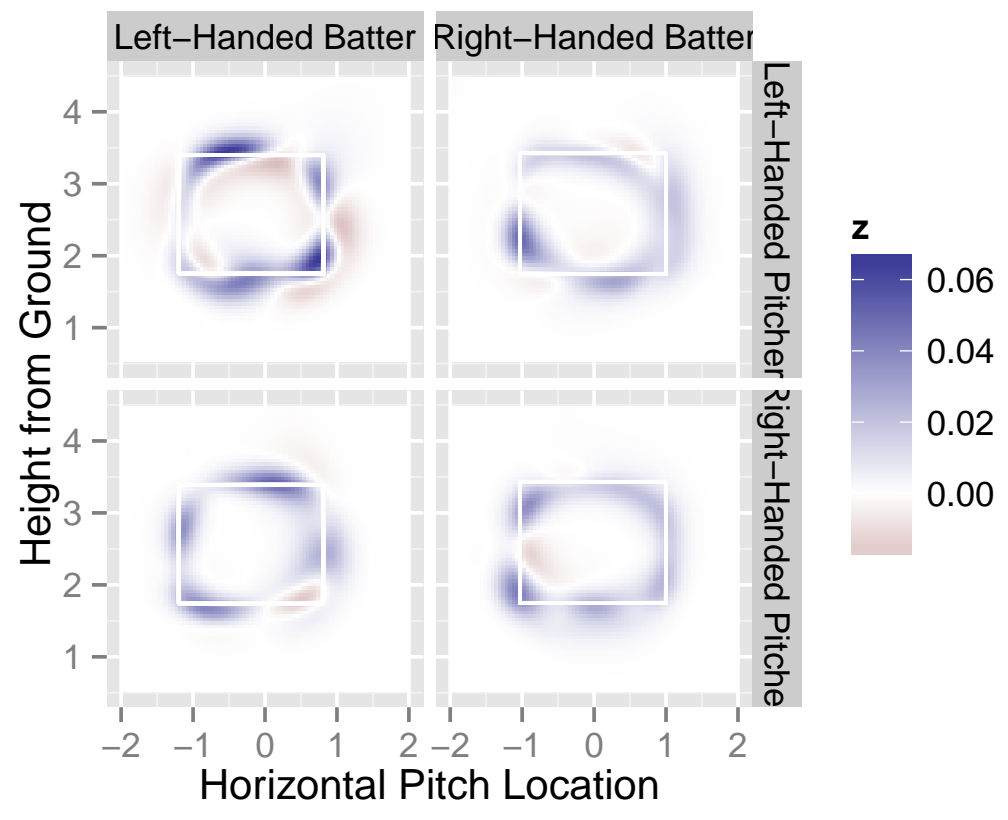

Figure 5: Difference between home and away pitchers in the probability of a strike (provided the umpire has to make a decision). The blue regions indicate a higher probability of a strike for home pitchers and red regions indicate a higher probability of a strike for away pitchers. Plots are faceted by the handedness of both the pitcher and the batter.

\section{D animation}

animateFX provides convenient and flexible functionality for animating the trajectory of any desired set of pitches. For demonstration purposes, this section animates every four-seam and cut fastball thrown by Mariano Rivera and Phil Hughes during the 2011 season. These pitches provide a good example of how facets play an important role in extracting new insights. Similar methods can be used to analyze any MLB player (or combination of players) in greater detail.

animateFX tracks three dimensional pitch locations over a sequence of two dimensional plots. The animation takes on the viewpoint of the umpire; that is, each time the plot refreshes, the balls are getting closer to the viewer. This is reflected with the increase in size of the points as the animation progresses. Obviously, some pitches travel faster than others, which explains the different sizes within a particular frame. Animations revert to the initial point of release once all of the baseballs have reached home plate. During an interactive session, animateFX produces a series of plots that may not viewed easily. One option available to the user is to wrap animation: : saveHTML around animateFX to view the animation in a browser with proper looping controls (Xie, 2013a).

To reduce the time and thinking required to produce these animations, animateFX has default settings for the geometry, color, opacity and size associated with each plot. Any of these assumptions can be altered - except for the point geometry. In order for animations to work, a data frame with the appropriately named PITCHf/x parameters (that is, $\mathrm{x} 0, \mathrm{y} 0, \mathrm{z} 0, \mathrm{vx} 0$, vy0, vz0, ax0, ay0 and az0) is required. In Figure 6, every four-seam and cut fastball thrown by Rivera and Hughes during the 2011 season is visualized using the pitches data frame obtained earlier (the animation is available at http://cpsievert.github.io/pitchRx/ani1).

animateFX(pitches, layer=list(theme_bw(), coord_equal(),

facet_grid(pitcher_name stand, labeller = relabel)))

In the animation corresponding to Figure 6, the upper right-hand portion (Rivera throwing to right-handed batters) reveals the clearest pattern in flight trajectories. Around the point of release, Rivera's two pitch types are hard to distinguish. However, after a certain point, there is a very different flight path among the two pitch types. Specifically, the drastic left-to-right movement of the cut fastball is noticeably different from the slight right-to-left movement of the four-seam fastball. In recent years, cut fastballs have gained notoriety among the baseball community as a coveted pitch for pitchers have at their disposal. This is largely due to the difficulty that a batter has in distinguishing the cut fastball from another fastball as the ball travels toward home plate. Clearly, this presents an advantage for the pitcher since they can use deception to reduce batter's ability to predict where the ball will cross home 


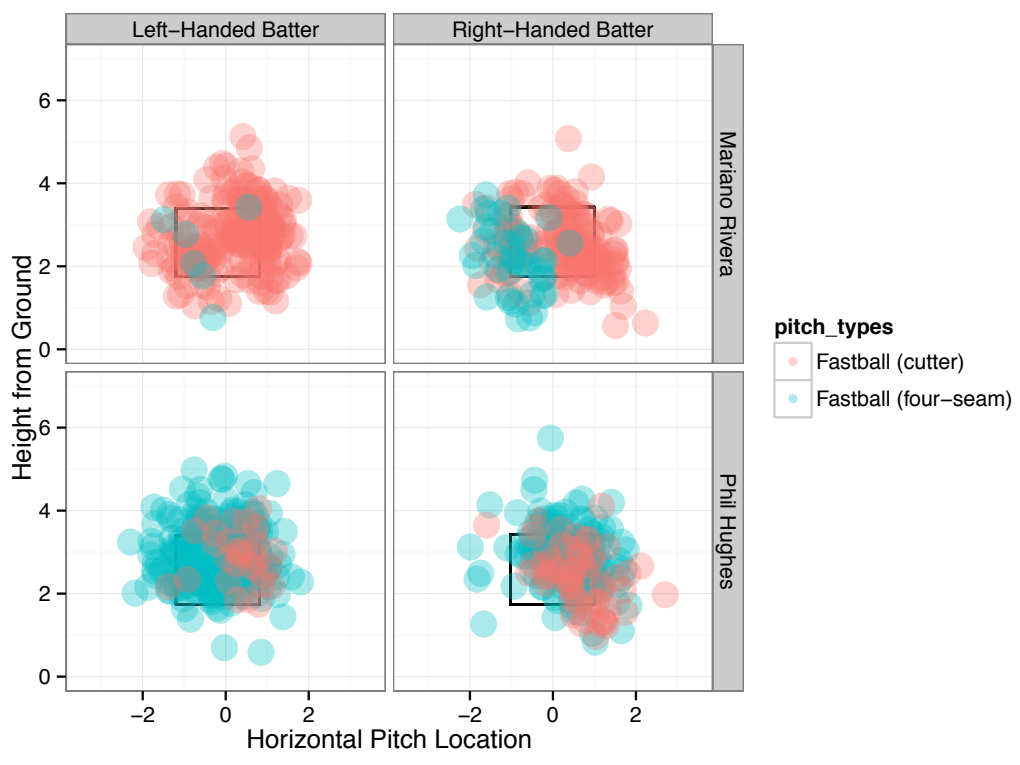

Figure 6: The last frame of an animation of every four-seam and cutting fastballs thrown by NY Yankee pitchers Mariano Rivera and Phil Hughes during the 2011 season. The actual animation can be viewed here. Pitches are faceted by pitcher and batting stance. For instance, the top left plot portrays pitches thrown by Rivera to left-handed batters.

plate. This deception factor combined with Rivera's ability to locate his pitches explain his accolades as one of the greatest pitchers of all time (Traub, 2010).

Although we see a clear pattern in Rivera's pitches, MLB pitchers are hardly ever that predictable. Animating that many pitches for another pitcher can produce a very cluttered graphic which is hard to interpret (especially when many pitch types are considered). However, we may still want to obtain an indication of pitch trajectory over a set of many pitches. A way to achieve this is to average over the PITCHf/x parameters to produce an overall sense of pitch type behavior (via the avg. by option). Note that the facet variables are automatically considered indexing variables. That is, in Figure 7 , there are eight 'average' pitches since there are two pitch types, two pitchers, and two types of batting stance (the animation is available at http://cpsievert.github.io/pitchRx/ani2).

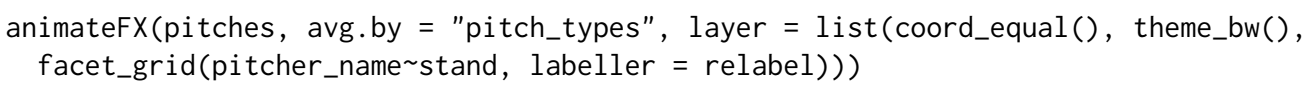

\section{Interactive 3D graphics}

rgl is an R package that utilizes OpenGL for graphics rendering. interactiveFX utilizes rgl functionality to reproduce flight paths on an interactive 3D platform. Figure 8 has two static pictures of Mariano Rivera's 2011 fastballs on this interactive platform. This is great for gaining new perspectives on a certain set of pitches, since the trajectories can be viewed from any angle. Figure 8 showcases the difference in trajectory between Rivera's pitch types.

Rivera <- subset(pitches, pitcher_name == "Mariano Rivera")

interactiveFX(Rivera, avg.by = "pitch_types")

\section{Conclusion}

pitchRx utilizes XML2R's convenient framework for manipulating XML content in order to provide easy access to PITCHf/ $x$ and related Gameday data. pitchRx removes access barriers which allows the average $\mathrm{R}$ user and baseball fan to spend their valuable time analyzing Gameday's enormous source of baseball information. pitchRx also provides a suite of functions that greatly reduce the amount of work involved to create popular PITCHf/x graphics. For those interested in obtaining other XML data, pitchRx serves as a nice example of leveraging XML2R to quickly assemble custom XML data collection mechanisms. 


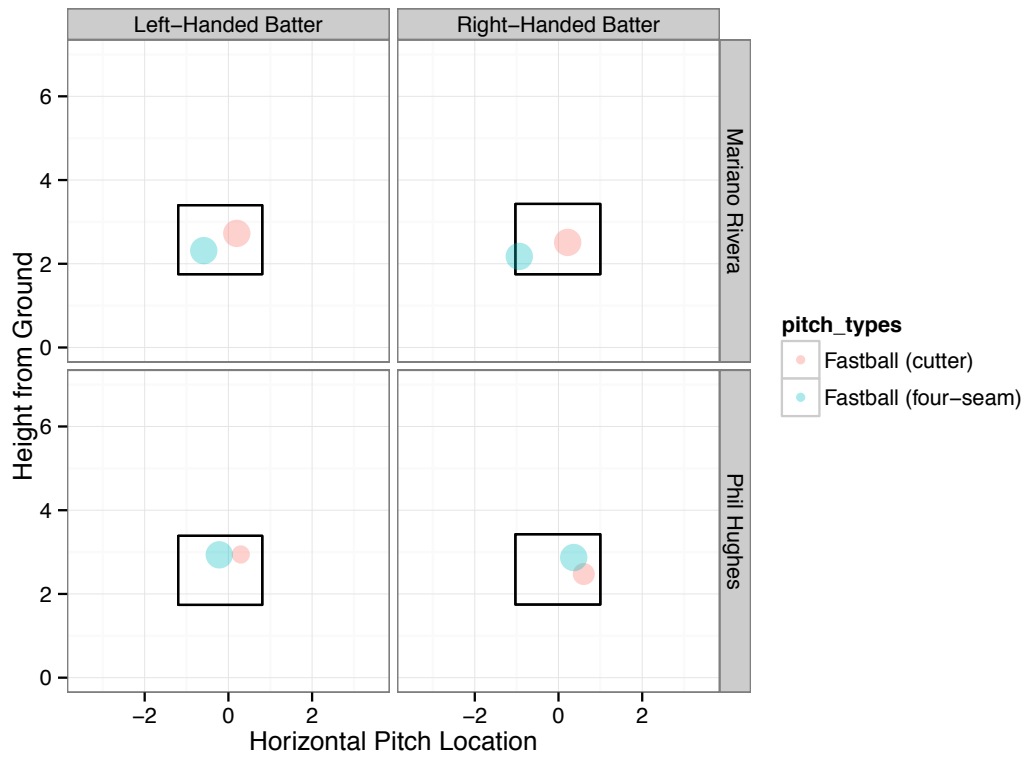

Figure 7: The last frame of an animation of 'averaged' four-seam and cutting fastballs thrown by NY Yankee pitchers Mariano Rivera and Phil Hughes during the 2011 season. The actual animation can be viewed here. PITCHf/x parameters are averaged over pitch type, pitcher and batting stance. For instance, the bottom right plot portrays an "average four-seam" and "average cutter" thrown by Hughes to right-handed batters.

\section{Acknowledgements}

Many thanks to my major professor, Dr. Heike Hofmann, for her direction and support throughout this project. Thanks also to the anonymous reviewers for helpful feedback. This document was created using the knitr package Xie (2013b). The source files can be found here.

\section{Bibliography}

D. Adler, D. Murdoch, and others. rgl: 3D Visualization Device System (OpenGL). URL http://rgl. neoscientists. org. R package version 0.93.963. [p6]

A. Alt and M. S. White. Tracking an object with multiple asynchronous cameras, 09 2008. URL http://www. patentlens.net/patentlens/patent/US_7062320/. [p5]

N. DiMeo. Baseball's particle accelerator, Aug. 2007. URL http://www. slate.com/articles/sports/ sports_nut/2007/08/baseballs_particle_accelerator. html. Last visited on 07/12/2012. [p5]

M. Fast. How to build a pitch database, Aug. 2007. URL http://fastballs. wordpress. com/2007/08/ 23/how-to-build-a-pitch-database/. Last visited on 12/15/2012. [p6]

M. Fast. Spinning yarn: A zone of their own, July 2011. URL http://www. baseballprospectus. com/ article. php?articleid=14572. Last visited on 06/17/2013. [p13]

E. Green and D. P. Daniels. What does it take to call a strike? three biases in umpire decision making. In MIT Sloan Sports Analytics Conference, 2014. [p12]

M. Marchi and J. Albert. Analyzing Baseball Data with R. Chapman and Hall/CRC, 2013. ISBN 9781466570221. URL http://baseballwithr.wordpress.com/. [p6]

B. Mills. Rethinking 'loess' for binomial-response PITCHf/x strike zone maps, Dec. 2010. URL http:// princeofslides.blogspot. com/2010/12/rethinking-loess-for-binomial-response.html. Last visited on 06/20/2013. [p14]

A. Nathan. A statistical study of PITCHf/x pitched baseball trajectories, Feb. 2008. URL http: //webusers.npl.illinois. edu/ a-nathan/pob/MCAnalysis. pdf. Last visited on 07/25/2012. [p5] 


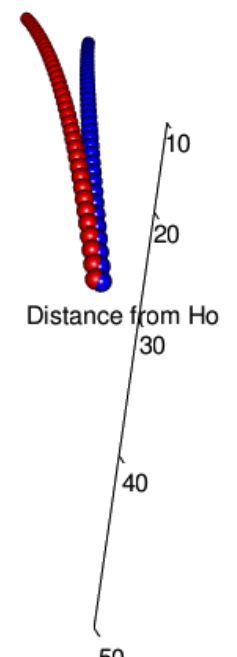

50

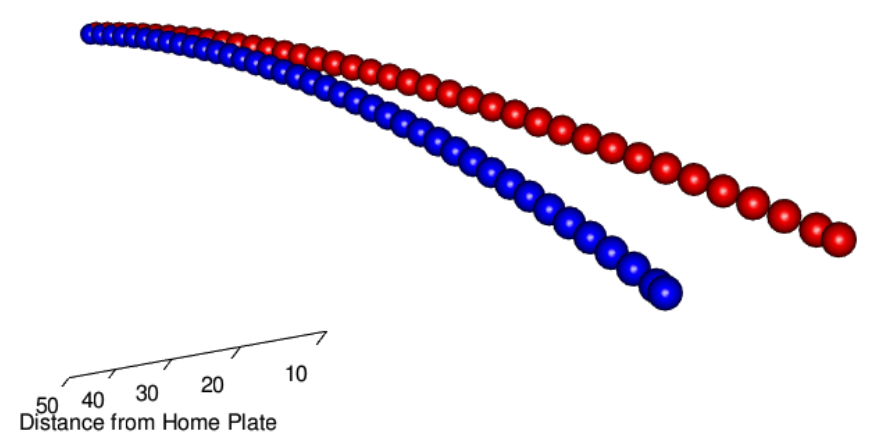

Distance from Home Plate

Figure 8: 3D scatterplot of pitches from Rivera. Pitches are plotted every one-hundredth of a second. Cutting fastballs are shown in red and four-seam fastballs are shown in blue. The left hand plot takes a viewpoint of Rivera and the right hand plot takes a viewpoint near the umpire. Note these are static pictures of an interactive object.

M. A. Pane, S. L. Ventura, R. C. Steorts, and A. C. Thomas. Trouble With The Curve: Improving MLB Pitch Classification. ArXiv e-prints, Apr. 2013. [p5]

R Special Interest Group on Databases. DBI: R Database Interface, 2013. URL http: //CRAN. R-project. org/package=DBI. R package version 0.2-7. [p11]

C. Sievert. XML2R: EasieR XML Data Collection, 2014a. URL http://cpsievert. gi thub.com/XML2R. R package version 0.0.4. [p6]

C. Sievert. pitchRx: Tools for Scraping XML Data and Visualizing Major League Baseball PITCHf/x, 2014b. URL http://cpsievert.github. com/pitchRx. R package version 1.5. [p5]

J. Traub. Mariano Rivera, king of the closers, June 2010. URL http://www. nytimes. com/2010/07/04/ magazine/04Rivera-t. html. Last visited on 02/04/2013. [p17]

H. Wickham. ggplot2: Elegant Graphics for Data Analysis. Springer, New York, 2009. ISBN 978-0-38798140-6. URL http://had.co.nz/ggplot2/book. [p6]

H. Wickham and R. Francois. dplyr: A Grammar of Data Manipulation, 2014. R package version 0.2. [p11]

S. Wood. Generalized Additive Models: An Introduction with R. Chapman and Hall/CRC, 2006. [p14]

Y. Xie. animation: An R package for creating animations and demonstrating statistical methods. Journal of Statistical Software, 53(1):1-27, 2013a. URL http: //www. jstatsoft. org/v53/i01/. [p16]

Y. Xie. Dynamic Documents with R and knitr. Chapman and Hall/CRC, 2013b. ISBN 978-1482203530. [p18]

Carson Sievert

Department of Statistics

Iowa State University

sievert@iastate.edu 